DOI: https://doi.org/10.24297/jap.v17i.8545

\title{
The Effects of Suction/Injection and Chemical Reaction on the Flow of Mass Transfer of a Micro Polar Fluid Past a Continuously Moving Plate in the Presence of Magnetic Field
}

\author{
S. S. Mustafa*, Rasha A. Mohamed \\ Department of Physics, Faculty of Education, Ain Shams University Roxy, Heliopolis, Cairo, Egypt \\ samiasayed@edu.asu.edu.eg
}

\begin{abstract}
An analysis is carried out to study the effects of chemical reaction and suction /injection on the flow of a micro polar fluid past a moving plate in the presence of magnetic field and mass transfer. The boundary layer equations are transformed to non-linear ordinary differential equations. Numerical results are presented for the distribution velocity, micro rotation profiles within the boundary layer. The effects of varying magnetic parameter, the non-dimensional chemical reaction parameter - . Schmidt number, porosity parameter, micro rotation parameter, and coupling constant parameter are revealed.
\end{abstract}

\section{Introduction}

Several engineering situations arise in which mass is transferred through a fluid that is in laminar flow including condensation sublimation evaporation on a plate or on the inner surface of the plate or on the inner surface of the tube. In some cases the surface of the plate or the tube may dissolve and then diffuse through the fluid boundary layer techniques have been shown to be very useful in gaining a better understanding of the above problems. One of the important engineering applications of the boundary layer equation is the case of a fluid passing over the surface of a solid with which it reacts.

In this case the reaction rate is both influenced by kinetic laws which govern the chemical reaction and the transport mechanisms induced by hydro magnetic equations. El Tawil et al [1], and Takhar et al [2] using numerical techniques such as finite difference or finite element have done a numerical study in the presence of chemical reaction. Approximate analytical solutions for the chemical reaction in isothermal laminar flow along a soluble flat plate, which is given by Hines et al [3].

In the previous studies the surface was assumed to be stationary and the fluid moves past this surface. We considered a moving surface, which is an important type of flow occurring in a number of technical processes. Examples may be found in continuous casting glass fiber production metal extrusion hot rolling the cooling and or drying of paper and textiles and wire drawing. The moving surface concept was introduced by Sakiadis [4].

The dynamics of micro polar fluids originated from the theory of Erigen [5], [6]. The micro polar fluids have been popular area of research the equations governing the flow of this type of fluids involve micro rotation vector and a gyration parameter. In addition to the classical velocity vector field this theory may be applied to explain the flow of colloidal solutions, liquid crystals, fluids with additives, suspension solutions, animal blood and many other situations. Peddieson and McNitt [7] have studied the boundary layer flow of such a micro polar fluid past a semi-infinite plate. On taking into account the gyration vector normal to the xy-plane and the micro inertia effects, the boundary layer flow of micro polar fluids past a semi-infinite plate was studied by Ahmadi [8].

Many others [9-14] studied the effect of suction /injection on the flow of a micro polar fluid past a continuously moving plate in the presence of radiation. In the present study we have analyzed the problem of the effect of the chemical reaction, suction/injection on a micro polar fluid past a continuously moving plate in the presence of uniform magnetic field. 


\section{Mathematical Formulation}

\begin{tabular}{|c|c|c|c|}
\hline \multicolumn{4}{|c|}{ Nomenclature } \\
\hline$f$ & Dimensionless stream function & $x$ & Distance along the surface \\
\hline $\mathrm{F}_{\mathrm{w}}$ & Porosity parameter & $y$ & Distance normal to the surface \\
\hline$g$ & Dimensionless micro rotation & c & Species concentration in the fluid \\
\hline G & Micro rotation parameter & $\mathrm{C}_{\infty}$ & $\begin{array}{l}\text { Species concentration with fluid away from the } \\
\text { plate }\end{array}$ \\
\hline$G_{1}$ & Micro rotation constant & $C_{w}$ & Species concentration near the plate \\
\hline$K$ & Coupling constant parameter & $D$ & Chemical molecular diffusivity \\
\hline$\kappa$ & Chemical reaction parameter & Sc & Schmidt number \\
\hline$K_{1}$ & Coupling constant & $n$ & Rate of chemical reaction \\
\hline $\operatorname{Re}$ & Reynolds number & $m$ & The magnetic constant \\
\hline$\sigma$ & Micro rotation component & $\psi$ & Stream function \\
\hline$u$ & Velocity in the $x$-direction & $\Phi$ & Dimensionless concentration function \\
\hline$U_{0}$ & Uniform velocity of the plate & $\gamma$ & Non-dimensional chemical reaction parameter \\
\hline $\mathrm{U}_{\infty}$ & Velocity in the ambient fluid & $\eta$ & Similarity variable \\
\hline$v$ & Velocity in the y-direction & $\mu$ & The dynamic viscosity \\
\hline$\kappa$ & Density of the fluid & $\alpha$ & Magnetic permeability \\
\hline$V_{w}$ & Wall suction/injection velocity & $B$ & Magnetic induction \\
\hline $\mathrm{S}$ & Constant characteristic of the fluid & $v$ & The kinematics viscosity \\
\hline
\end{tabular}

We consider a steady two-dimensional flow of a micro polar incompressible fluid past a continuously moving plate with suction or injection, chemical reaction under the influence of uniform magnetic field.

The $x$-axis is chosen along the plate and $y$-axis is taken normal to it. Under the usual boundary layer approximation, the flow and mass transfer in the presence of chemical reaction are governed by the following equation [9], [1]. 
And the boundary condition are given by

$$
\begin{aligned}
& \frac{\partial u}{\partial x}+\frac{\partial v}{\partial y}=0 \\
& u \frac{\partial u}{\partial x}+v \frac{\partial u}{\partial y}=v \frac{\partial^{2} u}{\partial y^{2}}+K_{1} \frac{\partial \sigma}{\partial y}=0 \\
& u=U_{0}, \quad v=\mathrm{V}_{\mathrm{w},} \quad c=\mathrm{C}_{\mathrm{w},}, \quad \sigma=0, \quad \text { At } y=0 \text {, } \\
& G_{1} \frac{\partial^{2} \sigma}{\partial y^{2}}-2 \sigma-\frac{\partial u}{\partial y}=0 \\
& y \rightarrow \infty, \quad u=0, \quad c=0, \quad \sigma=0 \\
& u \frac{\partial c}{\partial x}+v \frac{\partial c}{\partial y}=D \frac{\partial^{2} c}{\partial y^{2}}-\kappa c
\end{aligned}
$$

Here $u, v$ are the velocity components along $x, y$ coordinate respectively. $v=(\mu+s) / \rho$ is the apparent kinematics viscosity. $\mu$ the coeffiecient of dynamic viscosity. $s$ a constant characteristic of the fluid.

$\rho$ The density of the fluid. $\sigma$ The micro rotation component. $K_{1}=\mathrm{s} / \rho\left(K_{1}>0\right)$ the coupling constant. $\left(G_{1}>0\right)$ the micro rotation constant. c Species concentration in the fluid.

$\gamma$ Is the non-dimensional chemical reaction parameter. Sc Schmidt number, $\boldsymbol{C}_{\infty}$ is the species concentration away from the plate. $C_{w}$ is the species concentration near the plate. $U_{0}$ is the uniform velocity of the plate. $V_{w}$ a nondimensional velocity component at the wall.

The suitable similarity variables, for the problem under consideration, are [9]

$$
\begin{array}{ll}
\eta=y \sqrt{\frac{U_{0}}{2 v x}} & \psi=\sqrt{2 v U_{0} x} f(\eta) \\
u=\frac{\partial \psi}{c \partial y c_{\infty}}=U_{0} f^{\prime}(\eta) & v=-\frac{\partial \psi}{\sqrt{\frac{\partial x_{0}}{2 v x}} g(\eta)}-\frac{1}{2} \sqrt{\frac{2 v U_{0}}{x}}\left(f(\eta)-\eta f^{\prime}(\eta)\right) \\
c_{w}-c_{\infty} & \sigma=\sqrt{ }
\end{array}
$$

Where $f(\eta)$ is the dimensionless stream function.

Defining now the velocity components as

Where dashed mean differentiation with respect to $\eta$, the continuity equation is automatically satisfied, and the system of equation (2)-(4) becomes

$$
\begin{aligned}
& f^{\prime \prime \prime}(\eta)+f(\eta) f^{\prime \prime}(\eta)+K g^{\prime}(\eta)-2 m f^{\prime}(\eta)=0 \\
& G g^{\prime \prime}(\eta)-4 g(\eta)-2 f^{\prime \prime}(\eta)=0 \\
& \varphi^{\prime \prime}(\eta)+\operatorname{Sc} \varphi^{\prime}(\eta) f(\eta)-2 \gamma \operatorname{Re} \operatorname{Sc} \varphi(\eta)=0
\end{aligned}
$$

With the corresponding boundary condition.

$$
\begin{array}{llll}
f(0)=\mathrm{F}_{\mathrm{w},} & f(0)=1 \quad \phi(0)=1, & g(0)=0 \\
f(\infty)=0 & \phi(\infty)=0, \text { and } \quad g(\infty)=0 &
\end{array}
$$

In the previous equations, we have used, 
$K=K_{1} / \mathrm{v}$ (coupling constant parameter),

$G=G_{1} U_{0} / v x$ (micro rotation parameter),

$\mathrm{F}_{\mathrm{w}}=-\mathrm{V}_{\mathrm{w}}\left(2 x / v_{0}\right)^{1 / 2}$ (porosity parameter),

$\gamma=v \kappa / U_{0}^{2}$ (chemical reaction parameter),

$S c=v / D$ (Schmidt number).

The mass transfer parameter $F_{w}$ is positive for suction and negative for injection. The shear stress at the plate is given by.

$\tau_{\mathrm{w}}=(\mu+K)(\partial u / \partial y)_{y=0}+K(\sigma)_{y=0}$

The friction is given by

$C_{f}=\tau_{w} /(1 / 2) U_{0}^{2} \rho=-2 R e^{-(1 / 2)} f^{\prime}(0)$

\section{Numerical Solution}

The set of ordinary differential equations (8) - (10) with boundary condition (11) have been solved using finite difference method with $S c, F_{w}, k, m, G$ as prescribed parameters.

A program using symbolic and computational computer language (Mathematica 4.0) did the computations.

\section{Discussion of results}

Table 1 (the effect of porosity parameter on the plate friction $f^{\prime}(0)$ and plate couple stress $g^{\prime}(0)$ )

At $m=0.1, S c=1, y=0.1, G=2$, and $K=0.2$

\begin{tabular}{lll}
\hline$F_{w}$ & $f^{\prime}(0)$ & $g^{\prime}(0)$ \\
\hline-0.7 & -0.394919 & 0.258656 \\
-0.4 & -0.517365 & 0.299454 \\
-0.2 & -0.613720 & 0.327333 \\
0 & -0.721624 & 0.355257 \\
0.2 & -0.840479 & 0.382798 \\
0.4 & -0.969389 & 0.409554 \\
0.7 & -1.17911 & 0.447474 \\
\hline
\end{tabular}


Table 2 (the effect of magnetic parameter on the plate friction $f^{\prime}(0)$ and plate couple stress $g^{\prime}(0)$ )

At $F_{w}=0.4, S c=1, \gamma=0.1, G=2$, and $K=0.2$

\begin{tabular}{lll}
\hline$m$ & $f^{\prime}(0)$ & $g^{\prime}(0)$ \\
\hline 0 & -0.845661 & 0.386575 \\
0.1 & -0.969389 & 0.409554 \\
0.2 & -1.07739 & 0.428419 \\
0.3 & -1.17344 & 0.444237 \\
0.4 & -1.26013 & 0.457743 \\
\hline
\end{tabular}

Table 3 (the effect of magnetic parameter, chemical reaction parameter, Schmidt number on the surface mass transfer $\left.\ldots{ }^{\prime}(0)\right)$

At $F_{w}=0.4, G=2$, and $K=0.2$

\begin{tabular}{llllll}
\hline$m$ & $\gamma$ & $S c=0.1$ & $S c=0.5$ & $S c=1$ & $S c=1.5$ \\
\hline 0 & 0.2 & 0.26055 & 0.700393 & 1.11889 & 1.48655 \\
& 0.4 & 0.334191 & 0.854373 & 1.314 & 1.70198 \\
& 0.6 & 0.393945 & 0.977938 & 1.47197 & 1.87603 \\
& 0.8 & 0.445201 & 1.08344 & 1.60621 & 2.02266 \\
& 1 & 0.490662 & 1.17652 & 1.72355 & 2.14642 \\
1 & & & & & \\
& 0.2 & 0.245959 & 0.643325 & 1.02492 & 1.36198 \\
& 0.4 & 0.321642 & 0.810055 & 1.24058 & 1.60307 \\
& 0.6 & 0.382692 & 0.940148 & 1.4093 & 1.79114 \\
& 0.8 & 0.434865 & 1.04976 & 1.55034 & 1.94683 \\
2 & 1 & 0.481022 & 1.14573 & 1.67245 & 2.08008 \\
& & & & & \\
& 0.2 & 0.241166 & 0.62205 & 0.986636 & 1.30864 \\
& 0.4 & 0.317363 & 0.792827 & 1.20996 & 1.56029 \\
& 0.6 & 0.378746 & 0.925009 & 1.38262 & 1.75396 \\
& 0.8 & 0.431161 & 1.03597 & 1.52618 & 1.91327 \\
3 & 1 & 0.477506 & 1.1329 & 1.65008 & 2.04913 \\
& & & & & \\
& 0.2 & 0.238595 & 0.610021 & 0.964123 & 1.27649 \\
& 0.4 & 0.315024 & 0.782823 & 1.19158 & 1.53415 \\
& 0.6 & 0.376561 & 0.916063 & 1.36639 & 1.73099 \\
& 0.8 & 0.429087 & 1.02771 & 1.51133 & 1.89239 \\
& 1 & 0.475518 & 1.12514 & 1.63623 & 2.02976 \\
\hline
\end{tabular}


Table 4 (the effect of porosity parameter, chemical reaction parameter, Schmidt number on the surface mass transfer $\cdot \cdot(0))$

At $m=0.1, G=2$, and $K=0$.

\begin{tabular}{|c|c|c|c|c|c|}
\hline$F_{w}$ & $y$ & $\mathrm{Sc}=0.1$ & $S c=0.5$ & $\mathrm{Sc}=1$ & $\mathrm{Sc}=1.5$ \\
\hline \multirow[t]{5}{*}{-0.7} & 0.2 & 0.216361 & 0.430132 & 0.547744 & 0.612217 \\
\hline & 0.4 & 0.28879 & 0.588723 & 0.764596 & 0.873534 \\
\hline & 0.6 & 0.347689 & 0.715136 & 0.938211 & 1.08224 \\
\hline & 0.8 & 0.39831 & 0.823117 & 1.08579 & 1.2583 \\
\hline & 1 & 0.443283 & 0.918585 & 1.2152 & 1.41126 \\
\hline \multirow[t]{5}{*}{-0.4} & 0.2 & 0.226609 & 0.491193 & 0.671218 & 0.794185 \\
\hline & 0.4 & 0.299735 & 0.651463 & 0.889523 & 1.0564 \\
\hline & 0.6 & 0.35909 & 0.778703 & 1.06323 & 1.26382 \\
\hline & 0.8 & 0.410034 & 0.88707 & 1.21016 & 1.43746 \\
\hline & 1 & 0.455251 & 0.982662 & 1.33848 & 1.58741 \\
\hline \multirow[t]{5}{*}{-0.2} & 0.2 & 0.233807 & 0.535844 & 0.765384 & 0.937954 \\
\hline & 0.4 & 0.307307 & 0.696339 & 0.981918 & 1.19523 \\
\hline & 0.6 & 0.366911 & 0.823633 & 1.15407 & 1.39847 \\
\hline & 0.8 & 0.418036 & 0.93192 & 1.29944 & 1.56822 \\
\hline & 1 & 0.463389 & 1.02735 & 1.42621 & 1.71448 \\
\hline \multirow[t]{5}{*}{0} & 0.2 & 0.24136 & 0.583809 & 0.868926 & 1.09882 \\
\hline & 0.4 & 0.315149 & 0.743749 & 1.0815 & 1.34696 \\
\hline & 0.6 & 0.374952 & 0.870668 & 1.25079 & 1.54347 \\
\hline & 0.8 & 0.426223 & 0.978589 & 1.39372 & 1.70758 \\
\hline & 1 & 0.471688 & 1.07364 & 1.51826 & 1.84886 \\
\hline \multirow[t]{5}{*}{0.2} & 0.2 & 0.249317 & 0.635199 & 0.981535 & 1.27534 \\
\hline & 0.4 & 0.323301 & 0.793774 & 1.18809 & 1.5107 \\
\hline & 0.6 & 0.383247 & 0.919868 & 1.35326 & 1.69817 \\
\hline & 0.8 & 0.434626 & 1.02712 & 1.49287 & 1.85503 \\
\hline & 1 & 0.480177 & 1.12157 & 1.61454 & 1.99014 \\
\hline \multirow[t]{5}{*}{0.4} & 0.2 & 0.257724 & 0.690082 & 1.1027 & 1.46561 \\
\hline & 0.4 & 0.331802 & 0.846479 & 1.30139 & 1.6853 \\
\hline & 0.6 & 0.39183 & 0.971289 & 1.46128 & 1.86176 \\
\hline & 0.8 & 0.443277 & 1.07757 & 1.59674 & 2.00996 \\
\hline & 1 & 0.488883 & 1.1712 & 1.71493 & 2.13784 \\
\hline \multirow[t]{5}{*}{0.7} & 0.2 & 0.27125 & 0.778967 & 1.29897 & 1.77199 \\
\hline & 0.4 & 0.34527 & 0.930647 & 1.4831 & 1.96459 \\
\hline & 0.6 & 0.405302 & 1.05268 & 1.63315 & 2.12177 \\
\hline & 0.8 & 0.456771 & 1.15692 & 1.76101 & 2.25486 \\
\hline & 1 & 0.502401 & 1.24888 & 1.87291 & 2.37025 \\
\hline
\end{tabular}


$\underline{\text { Table } 5}$ (the effect of coupling constant parameter on the plate friction $f^{\prime}(0)$, plate couple stress $g^{\prime}(0)$ and surface mass transfer $\left.\cdot \cdot^{\prime}(0)\right)$

At $m=0.1, F_{\mathrm{w}}=0.4, \mathrm{Sc}=1, y=0.1$, and $G=2$

\begin{tabular}{llll}
\hline$K$ & $\left.\cdot \cdot^{\prime}(0)\right)$ & $f^{\prime}(0)$ & $g^{\prime}(0)$ \\
\hline 0 & 0.980804 & -0.980804 & 0.406405 \\
0.2 & 0.978761 & -0.969389 & 0.409554 \\
0.4 & 0.976540 & -0.957838 & 0.412893 \\
0.6 & 0.974119 & -0.946154 & 0.416442 \\
0.8 & 0.971471 & -0.934346 & 0.420226 \\
1 & 0.968561 & -0.922423 & 0.424274 \\
\hline
\end{tabular}

Table 6 (the effect of microrotation parameter on the plate friction $f^{\prime \prime}(0)$, plate couple stress $g^{\prime}(0)$ and surface mass transfer $\left.\cdot \cdot^{\prime}(0)\right)$.

At $m=0.1, \mathrm{~F}_{\mathrm{w}}=0.4, \mathrm{Sc}=1, \gamma=0.1$, and $K=0.2$

\begin{tabular}{llll}
\hline$G$ & $\left.\cdot \cdot^{\prime}(0)\right)$ & $F^{\prime \prime}(0)$ & $g^{\prime}(0)$ \\
\hline 0.5 & & & \\
\hline 1 & 0.9768630 & -0.9650640 & 0.8960680 \\
1.5 & 0.9778250 & -0.9668610 & 0.6262110 \\
2 & 0.9783840 & -0.9682830 & 0.4920490 \\
2.5 & 0.9787610 & -0.9693890 & 0.4095540 \\
\hline
\end{tabular}


Table 7 (the effect of micro rotation parameter on the $c$

oncentration $\varphi(\underline{\eta}))$.

At $\mathrm{m}=0.1, \mathrm{~F}_{\mathrm{w}}=0.4, \mathrm{Sc}=1, \gamma=0.1$, and $\mathrm{K}=0.2$

\begin{tabular}{|l|l|l|l|l|}
\hline \multirow{2}{*}{$\eta$} & \multicolumn{5}{|c|}{$\phi$} \\
\cline { 2 - 5 } & G=2 & G=3 & G=4 & \\
\hline 0.4 & 0.4837087 & 0.4836693 & 0.4836468 & 0.4836323 \\
\hline 0.8 & 0.2252708 & 0.2252001 & 0.2251581 & 0.2251302 \\
\hline 1.2 & 0.1024238 & 0.1023472 & 0.1023002 & 0.1022682 \\
\hline 1.6 & 0.0458957 & 0.0458325 & 0.0457926 & 0.0457649 \\
\hline 2 & 0.0203905 & 0.0203464 & 0.0203180 & 0.0202979 \\
\hline 2.4 & 0.0090148 & 0.0089874 & 0.0089693 & 0.0089564 \\
\hline 2.8 & 0.0039747 & 0.0039588 & 0.0039482 & 0.0039405 \\
\hline 3.2 & 0.0017498 & 0.0017412 & 0.0017353 & 0.0017310 \\
\hline 3.6 & 0.0007697 & 0.0007652 & 0.0007621 & 0.0007598 \\
\hline 4 & 0.0003385 & 0.0003362 & 0.0003346 & 0.0003334 \\
\hline 4.4 & 0.0001488 & 0.0001477 & 0.0001468 & 0.0001462 \\
\hline 4.8 & 0.0000654 & 0.0000648 & 0.0000645 & 0.0000642 \\
\hline 5.2 & 0.0000288 & 0.0000285 & 0.0000283 & 0.0000281 \\
\hline 5.6 & 0.0000126 & 0.0000125 & 0.0000124 & 0.0000123 \\
\hline
\end{tabular}

$\underline{\text { Table } 8}$ (the effect of coupling constant parameter on the concentration $\varphi(\eta)$ ).

At $m=0.1, F_{\mathrm{w}}=0.4, \mathrm{Sc}=1, \gamma=0.1$, and $G=2$

\begin{tabular}{|l|l|l|l|l|l|}
\hline \multirow{2}{*}{$\eta$} & \multicolumn{5}{|l|}{} \\
\cline { 2 - 6 } & $K=0.2$ & $K=0.4$ & $K=0.6$ & $K=0.8$ & $K=1$ \\
\hline 0.4 & 0.4837087 & 0.4838254 & 0.4839544 & 0.4840972 & 0.4842560 \\
\hline 0.8 & 0.2252708 & 0.2255256 & 0.2258016 & 0.2261014 & 0.2264282 \\
\hline 1.2 & 0.1024238 & 0.1027395 & 0.1030788 & 0.1034448 & 0.1038410 \\
\hline
\end{tabular}




\begin{tabular}{|l|l|l|l|l|l|}
\hline 1.6 & 0.0458957 & 0.0461841 & 0.0464937 & 0.0468270 & 0.0471871 \\
\hline 2 & 0.0203905 & 0.0206093 & 0.0208444 & 0.0210977 & 0.0213718 \\
\hline 2.4 & 0.0090148 & 0.0091617 & 0.0093199 & 0.0094909 & 0.0096764 \\
\hline 2.8 & 0.0039747 & 0.0040652 & 0.0041631 & 0.0042693 & 0.0043851 \\
\hline 3.2 & 0.0017498 & 0.0018023 & 0.0018594 & 0.0019216 & 0.0019897 \\
\hline 3.6 & 0.0007697 & 0.0007989 & 0.0008306 & 0.0008655 & 0.0009039 \\
\hline 4 & 0.0003385 & 0.0003541 & 0.0003712 & 0.0003901 & 0.0004110 \\
\hline 4.4 & 0.0001488 & 0.0001569 & 0.0001659 & 0.0001759 & 0.0001870 \\
\hline 4.8 & 0.0000654 & 0.0000696 & 0.0000742 & 0.0000793 & 0.0000851 \\
\hline 5.2 & 0.0000288 & 0.0000308 & 0.0000332 & 0.0000358 & 0.0000388 \\
\hline 5.6 & 0.0000126 & 0.0000137 & 0.0000148 & 0.0000161 & 0.0000176 \\
\hline
\end{tabular}

Table 1 indicates the effect of mass transfer $F_{w}$ on the plate friction and plate couple stress. We observe that injection reduces the friction factor as well as plate couple stress whereas the suction has the opposite effect.

Table 2 indicates the effect of the magnetic parameter on the plate friction and plate couple stress. The magnetic parameter decreases the plate friction and increases the plate couple stress.

Table 3 displays the effect of the magnetic parameter $m$, the chemical reaction parameter $\gamma$, and Schmidt number Sc on the fluid surface mass transfer. We note that the surface mass transfer decreases with the magnetic parameter. It increases with the chemical reaction parameter and increases with the Schmidt number.

Table 4 displays the effect of suction/injection on the surface mass transfer. We note that suction decreases the surface mass transfer while the injection has an opposite effect.

Table 5 indicates the effect of the coupling stress parameter on the plate friction, plate couple stress and surface mass transfer. The coupling stress parameter increases the plate friction, decreases the plate couple stress and decreases the surface mass transfer

Table 6 indicates the effect of the micro rotation parameter on the plate friction, plate couple stress and surface mass transfer. The micro rotation parameter decreases the plate friction, decreases the plate couple stress and increases the surface mass transfer.

Table 7 indicates the effect of the micro rotation parameter on the concentration. The micro rotation parameter decreases the concentration.

Table 8 indicates the effect of the coupling constant parameter on the concentration. The coupling constant parameter increases the concentration. 


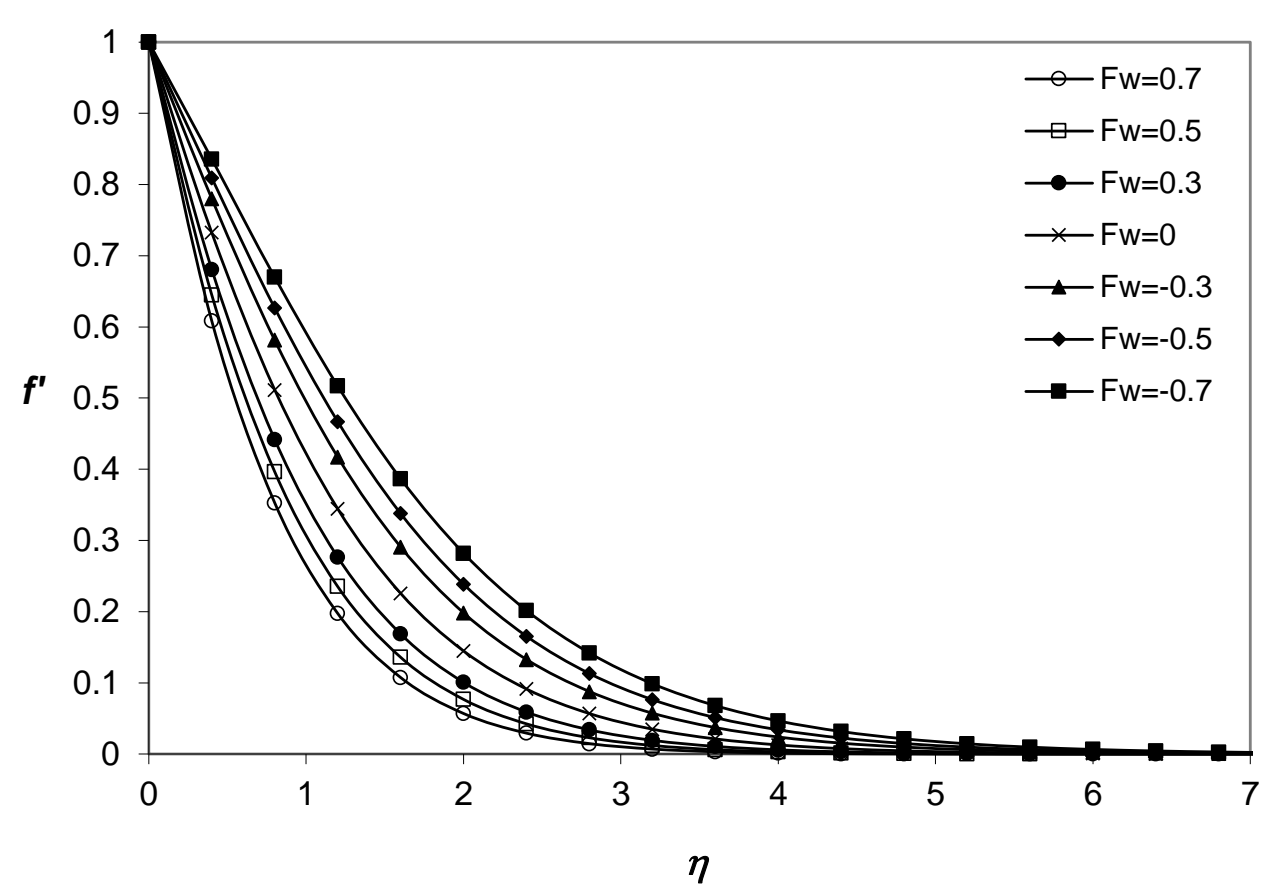

Fig.(1): Velocity profiles for various values of mass transfer (At $m=0.1, S c=1, \gamma=0.1, G=2$, and $K=0.2$ )

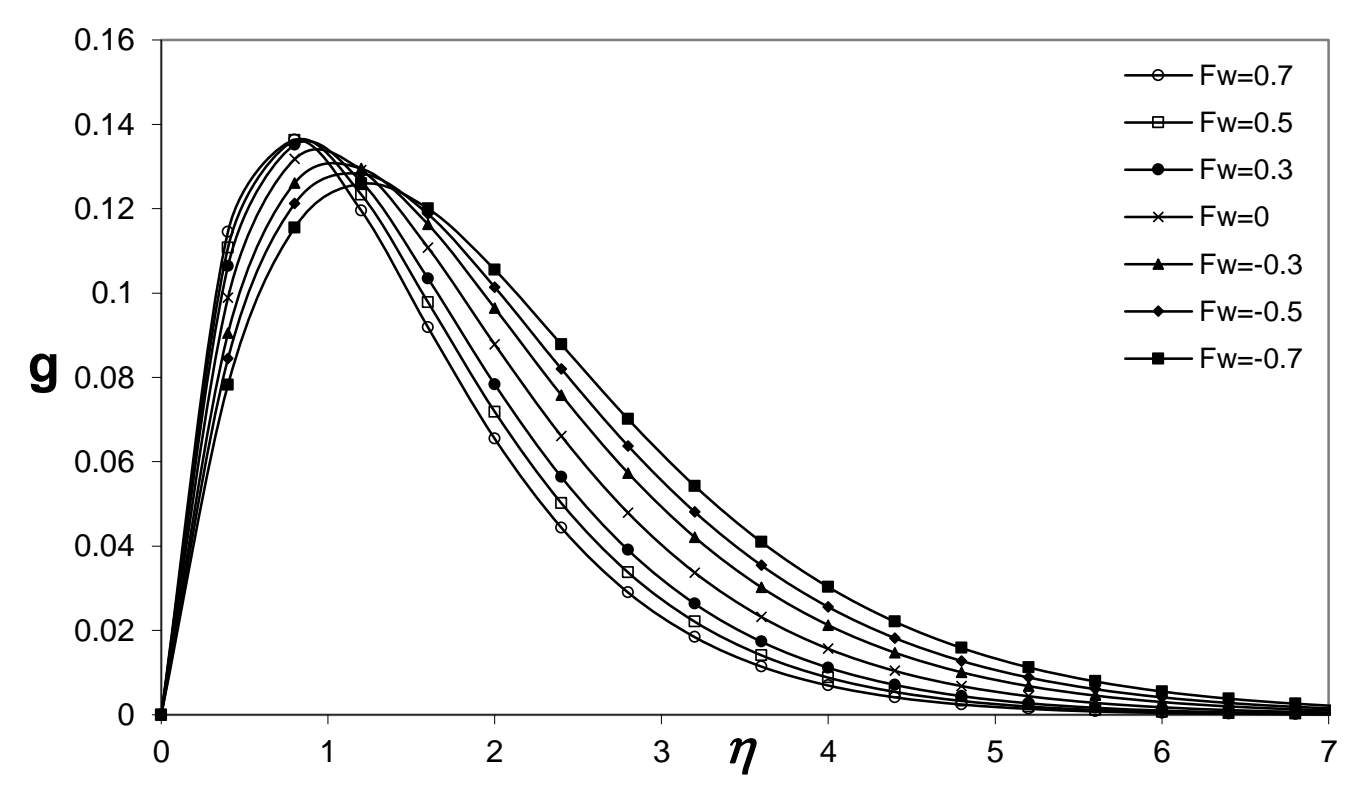

Fig.(2): Micro rotation profiles for various values of mass transfer (At $m=0.1, S c=1, y=0.1, G=2$, and $K=0.2$ ) 


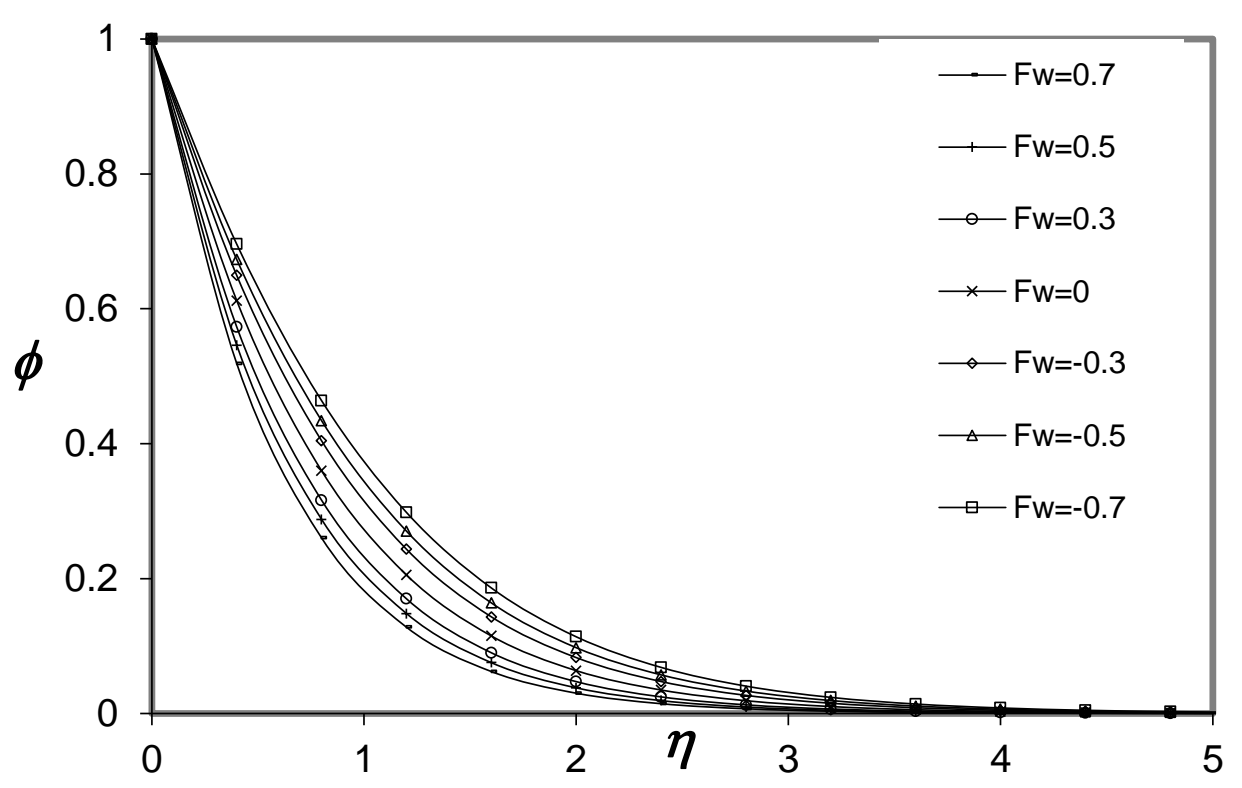

Fig.(3): Concentration profiles for various values of mass transfer (At $m=0.1, S c=1, \gamma=0.1, G=2$, and $K=0.2$

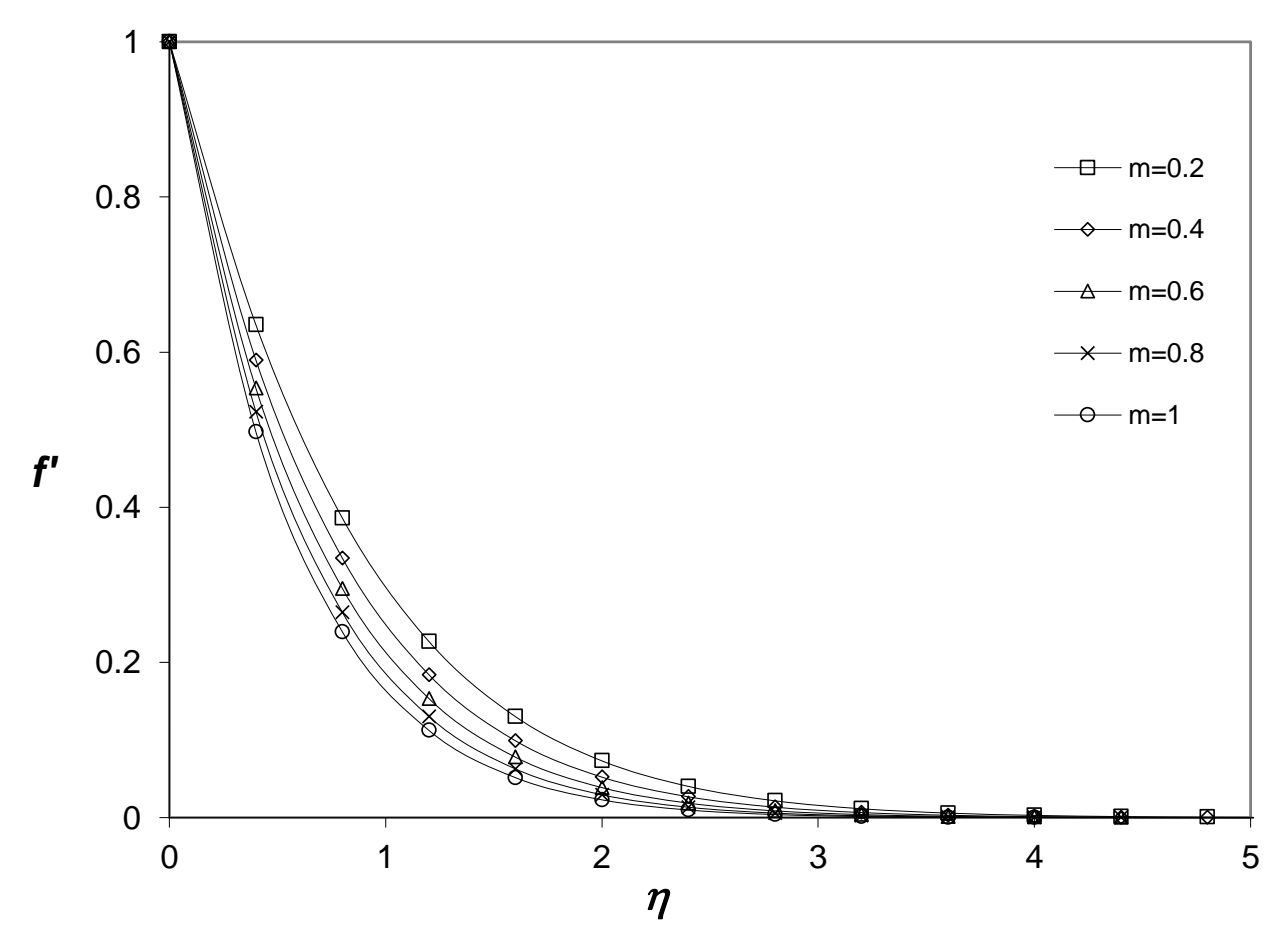

.Fig.(4): Velocity profiles for various values of magnetic parameter.

$$
\text { (At } F_{w}=0.4, S c=1, Y=0.1, G=2 \text {, and } K=0.2 \text { ) }
$$




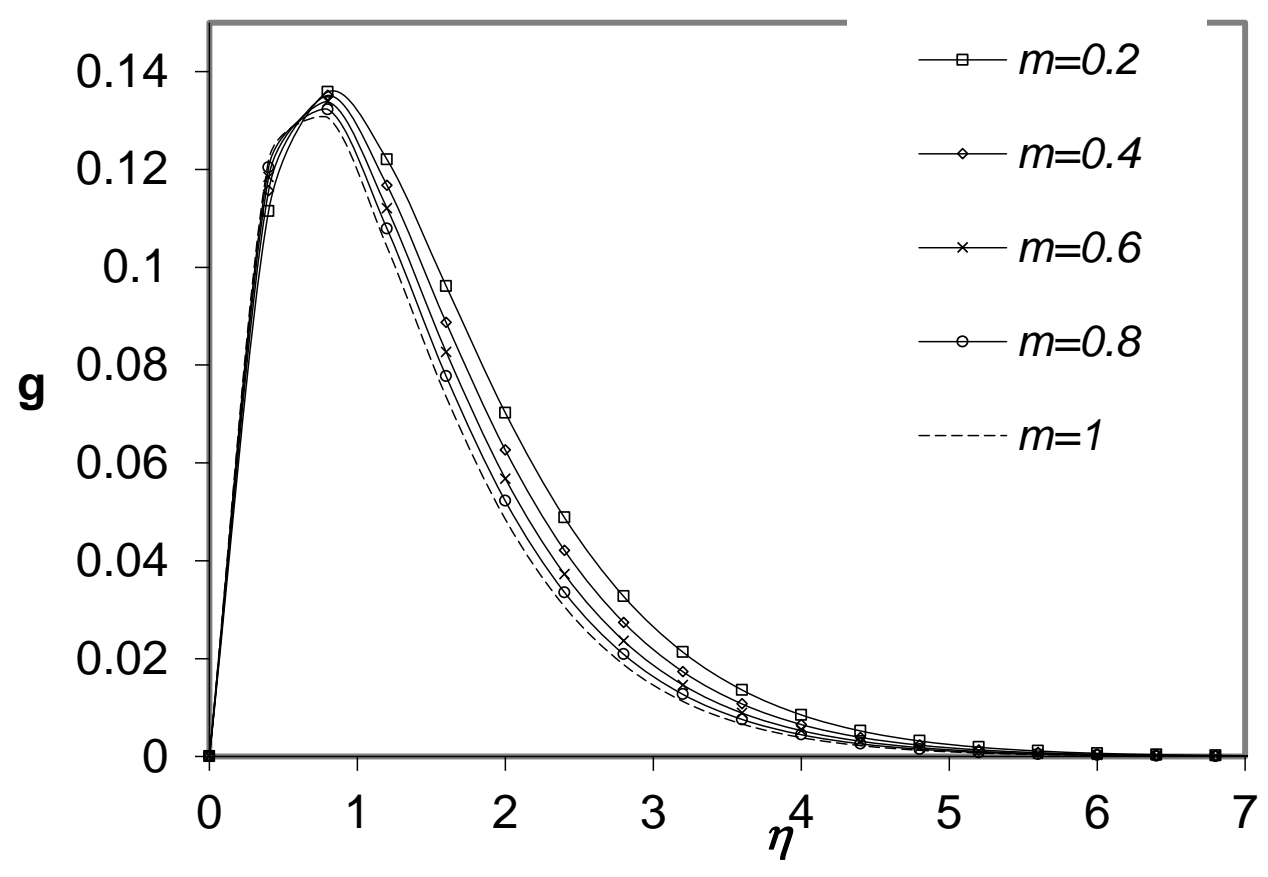

Fig.(5): Microrotation profiles for various values of magnetic parameter.

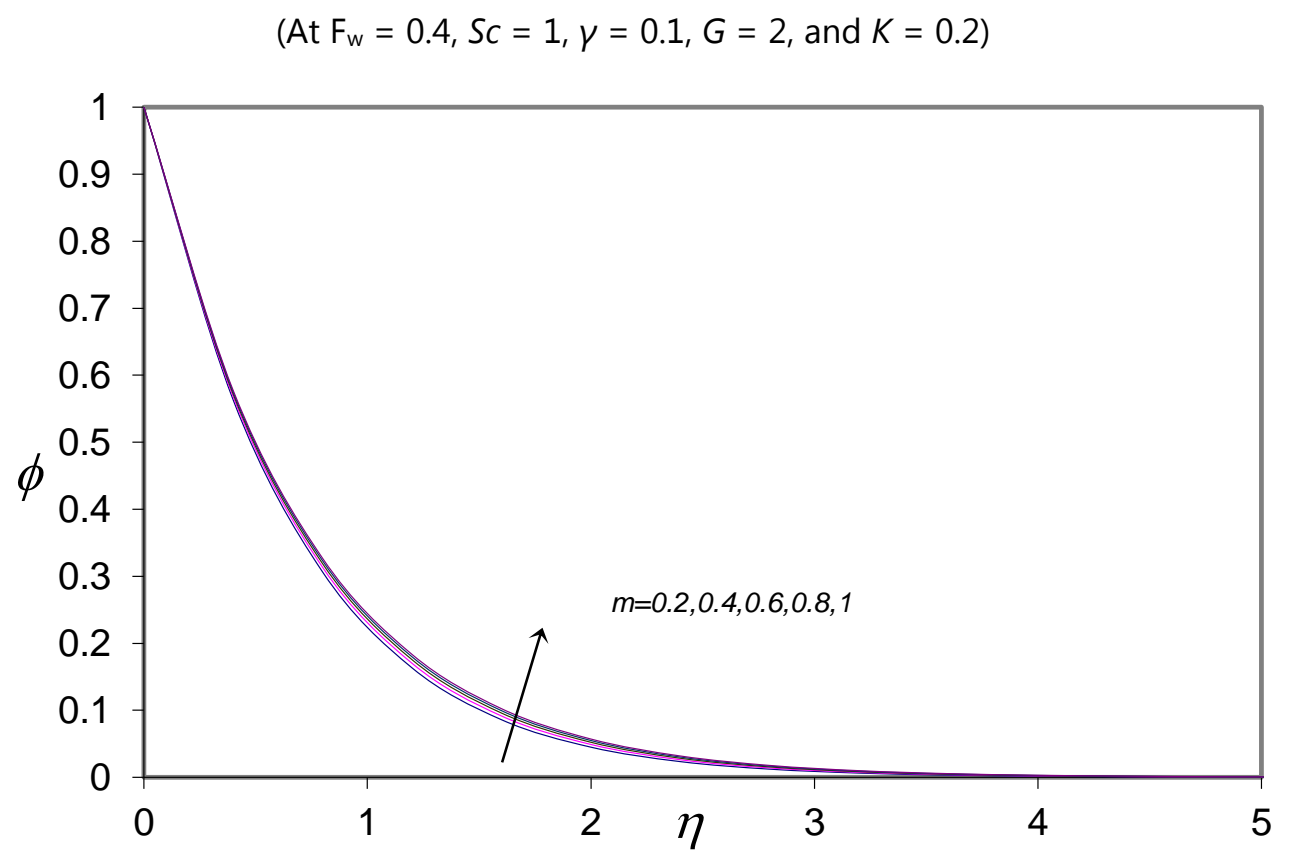

Fig.(6): Concentration profiles for various values of magnetic parameter.

$$
\text { (At } F_{w}=0.4, S c=1, Y=0.1, G=2 \text {, and } K=0.2 \text { ) }
$$




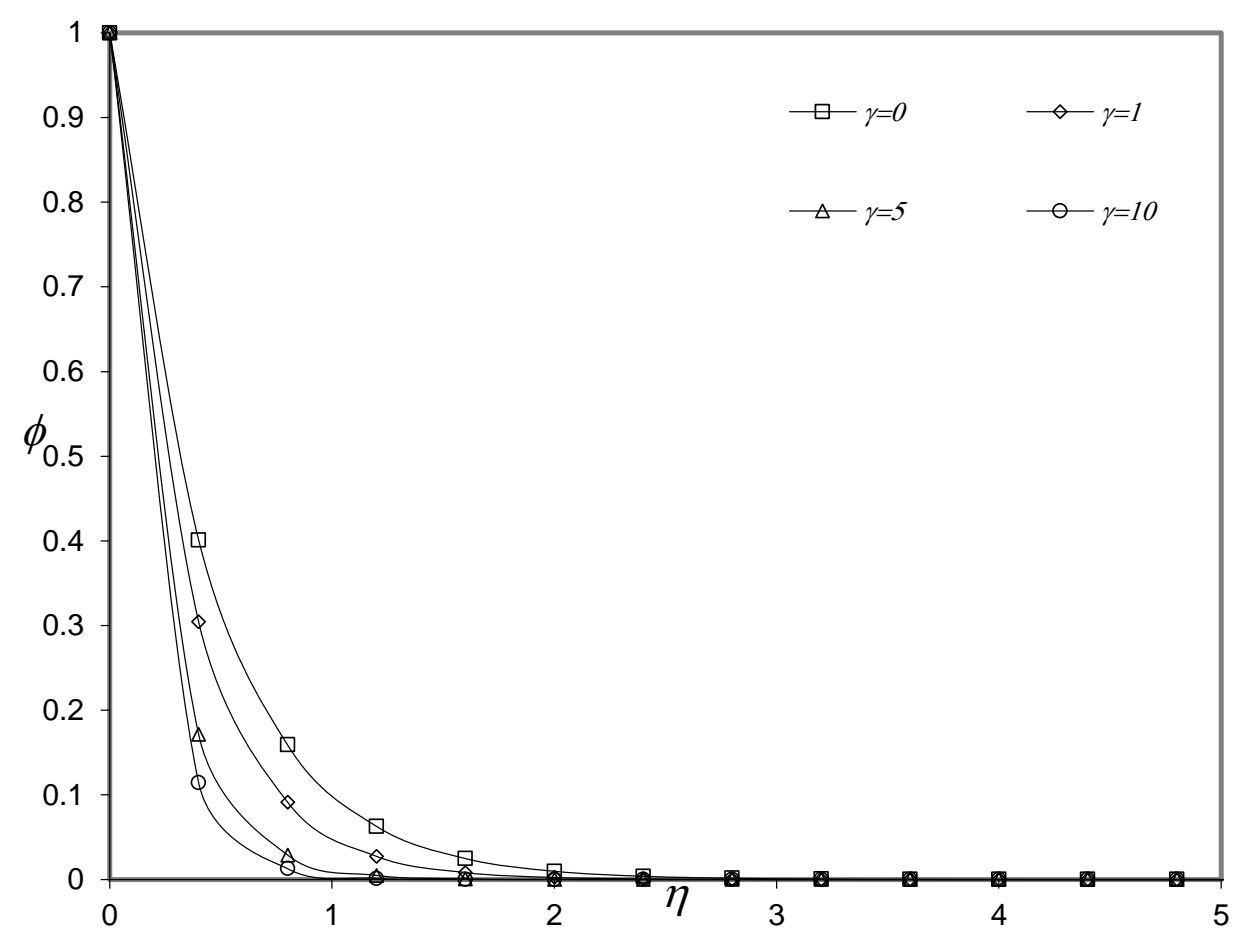

Fig.(7): Concentration profiles for various values of chemical reaction parameter.

$$
\text { (At } F_{\mathrm{w}}=0.4, S c=1, m=0.1, G=2 \text {, and } K=0.2 \text { ) }
$$

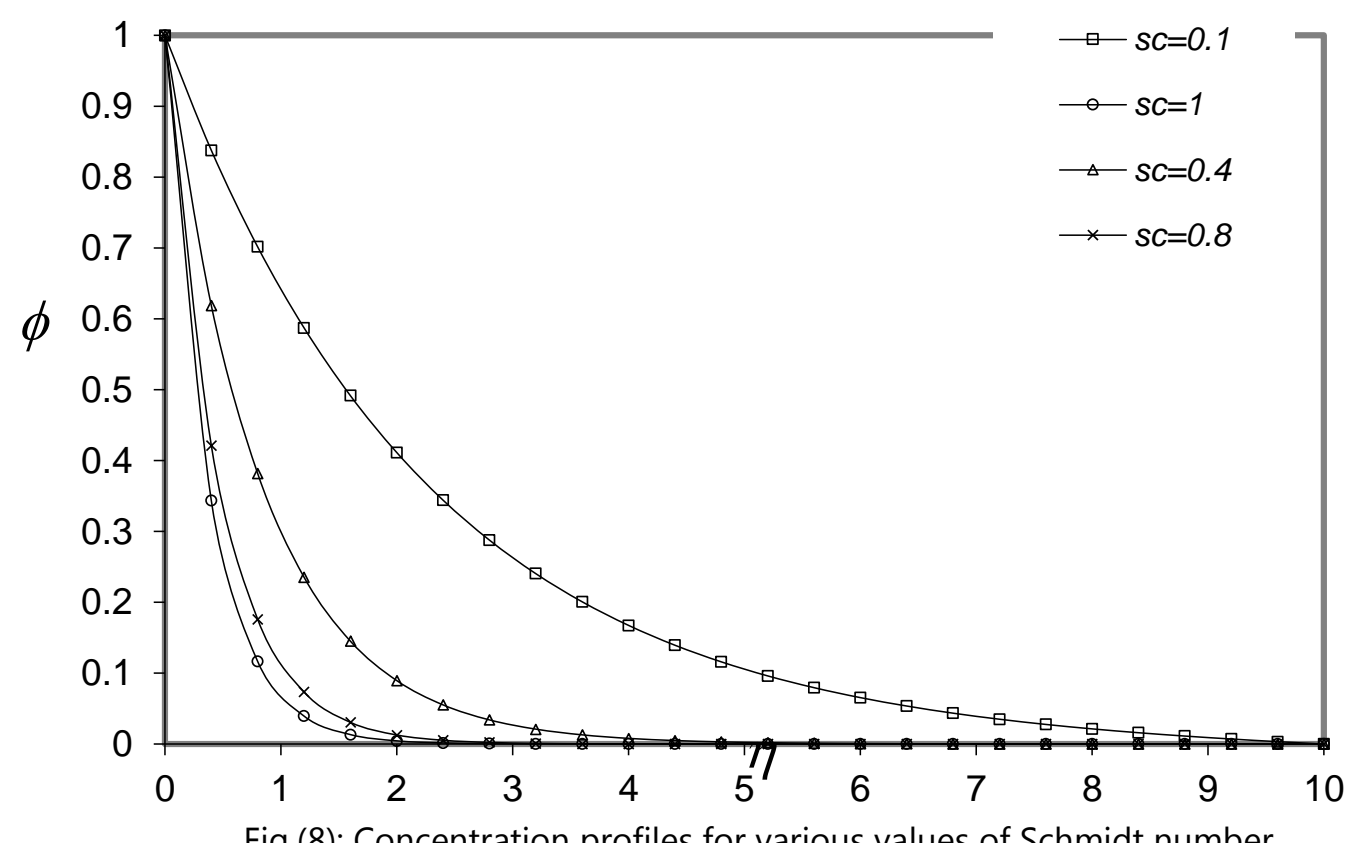

Fig.(8): Concentration profiles for various values of Schmidt number.

(At $F_{w}=0.4, m=0.1, y=0.1, G=2$, and $K=0.2$ ) 


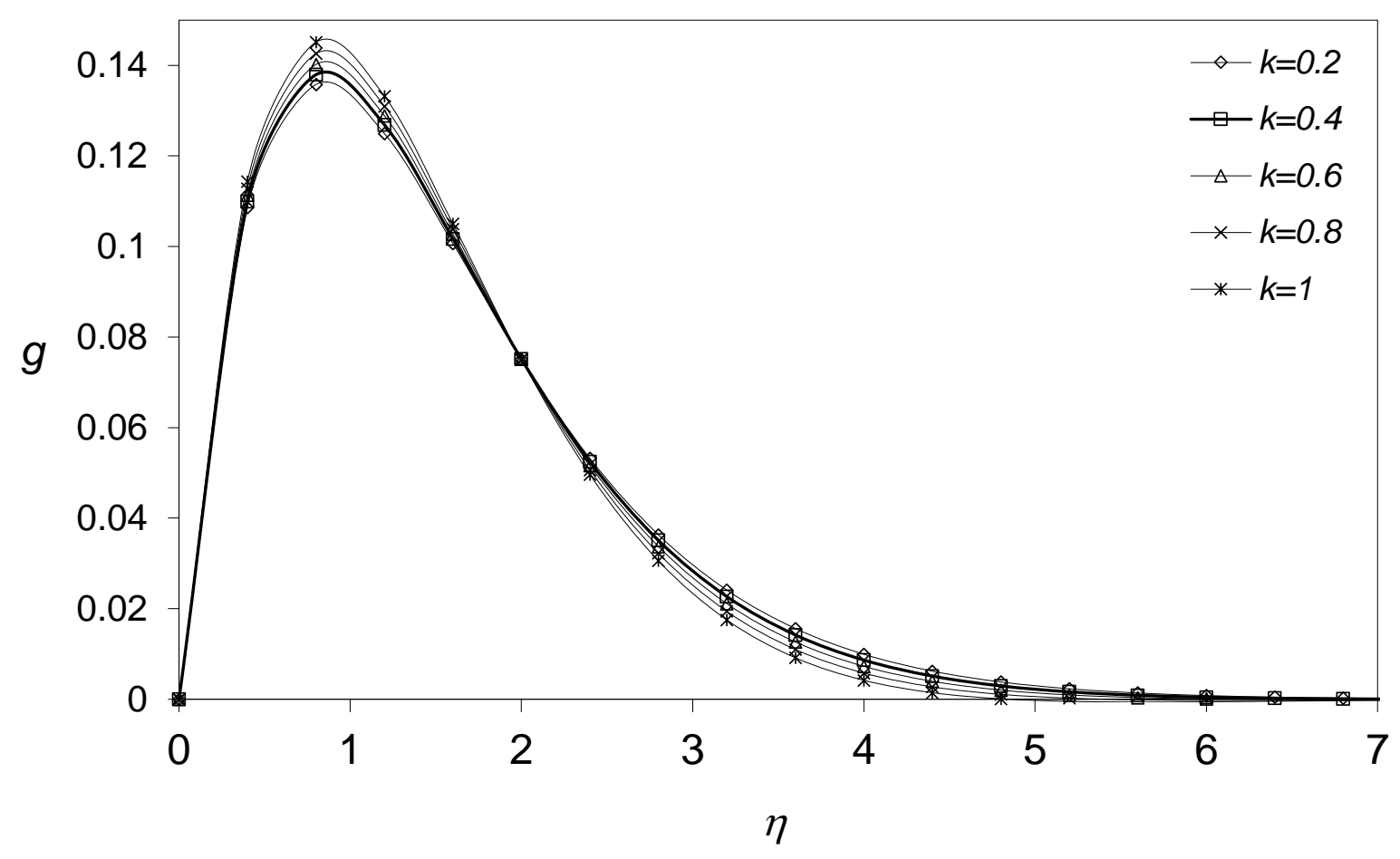

Fig.(9): Micro rotation profiles for various values of coupling constant parameter.

$$
\text { (At } F_{\mathrm{w}}=0.4, S c=1, y=0.1, G=2 \text {, and } m=0.1 \text { ) }
$$

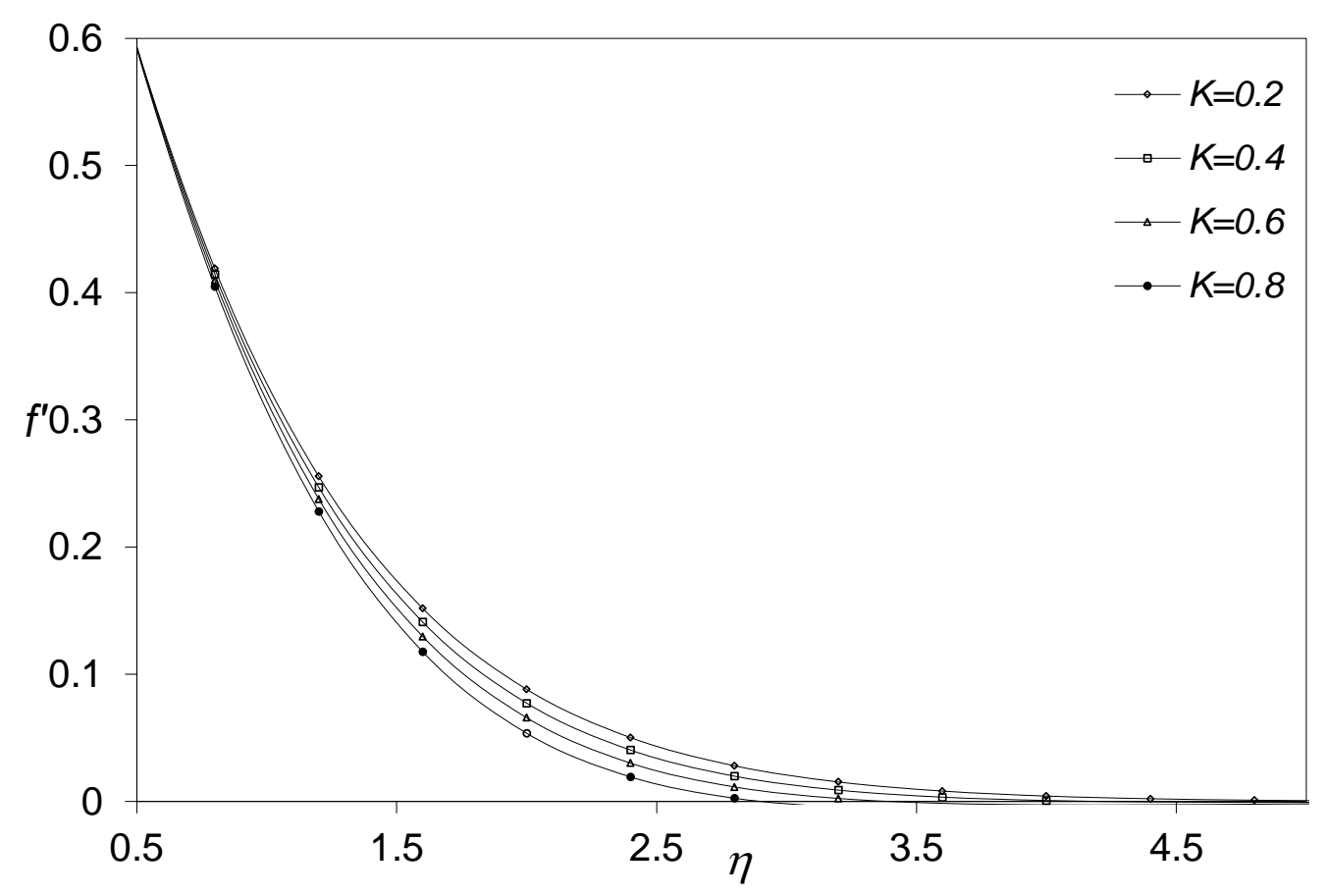

Fig.(10): Velocity profiles for various values of coupling constant parameter.

$$
\text { (At } F_{w}=0.4, S c=1, y=0.1, G=2 \text {, and } m=0.1 \text { ) }
$$




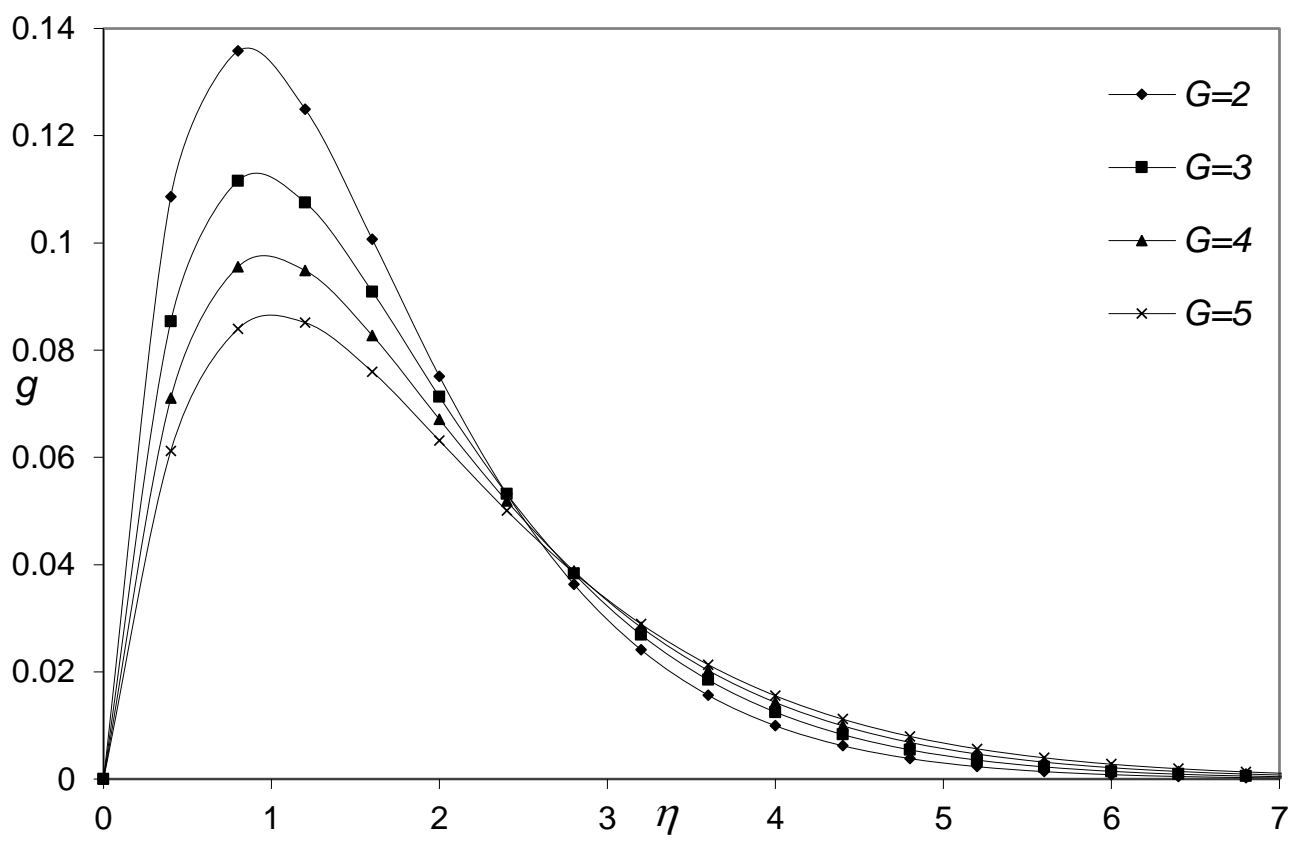

Fig.(11): Micro rotation profiles for various values of micro rotation parameter.

$$
\text { (At } \mathrm{F}_{\mathrm{w}}=0.4, \mathrm{Sc}=1, \gamma=0.1, m=0.1 \text {, and } K=0.2 \text { ) }
$$

Fig.1 indicates the velocity profiles showing the effect of $F_{w}$ it can be seen that velocity increases with injection and decreases with increases in suction.

Fig. 2 indicates the micro rotation profiles showing effect of $F_{w}$. The micro rotation reaches a maximum and then decay to zero. Increasing values of the injection parameter move the location of the maximum value of the micro rotation away from the surface. The locations of the maximum value of the micro rotation profiles, as a whole, are less with increases in suction.

Fig.3 indicates the concentration profiles showing the effect of $F_{w_{1}}$ it can be seen that concentration increases with injection and decreases with increases in suction.

Fig.4 indicates the velocity profiles showing the effect of magnetic parameter, it can be seen that velocity decreases with increases in magnetic parameter.

Fig.5 indicates the micro rotation profiles showing effect of magnetic parameter. The micro rotation reaches a maximum and then decay to zero. Increasing value of the magnetic parameter decreases the maximum value of the micro rotation profiles.

Fig.6 indicates the concentration profiles showing the effect of magnetic parameter, with increases in magnetic parameter. It can be seen that concentration increases

Fig.7 indicates the concentration profiles showing the effect of chemical reaction parameter, it can be seen that the concentration decreases with increases in chemical reaction parameter.

Fig. 8 indicates the concentration profiles showing the effect of Schmidt number, it can be seen that the concentration decreases with increases in Schmidt number. 
Fig. 9 indicates the micro rotation profiles showing effect of coupling constant parameter. The micro rotation reaches a maximum and then decay to zero. Increasing value of the coupling constant parameter decreases the maximum value of the micro rotation profiles.

Fig.10 indicates the velocity profiles showing the effect of coupling constant parameter, it can be seen that the velocity decreases with increases in coupling constant parameter.

Fig. 11 indicates the micro rotation profiles showing effect of micro rotation parameter. The micro rotation reaches a maximum and then decay to zero. Increasing value of the micro rotation parameter decreases the maximum value of the micro rotation profiles.

\section{Conclusion}

In this paper, we have extended the problem of flow of a micro polar fluid past a continuously moving plate in the presence of suction/injection that studied by El-Arabawy A. M. [12], to include the effects of mass transfer with chemical reaction in the presence of magnetic field.

The presence of mass transfer with chemical reaction and magnetic field introduce three extra parameter into the problem, namely $S c$ (Schmidt number), $\gamma$ (chemical reaction parameter), $m$ (magnetic parameter). The effects of the magnetic parameter, Schmidt number, and the chemical reaction parameter on the mass transfer rate, the flow, and the concentration profiles are revealed. The results show that the mass transfer rate increases with the magnetic parameter, it decreases with the Schmidt number and the chemical reaction parameter.

\section{References}

1. El-Tawil, M. A, Hassanien, I. A, the computer simulation study of the stochastic chemical reaction in a laminar boundary layer flow over a stretching surface, J. inst. Math. \& Comp. Sci, 6(1995) 105-120.

2. Takhar, H. S, Chamkha, A. J, Nath, G, flow and mass transfer on a stretching sheet with a magnetic field and chemically reactive species, Int. J. Eng. Sci, 38(2000) 1303-1314.

3. Hines, A. L, and Maddox, R. N, Mass transfer fundamentals and application, Prentice Hall, New Jersey (1985).

4. Sakiadis, B. C, Boundary layer behavior on continuos solid flat surface, Am. ICHE. J, 7 (1961) 221.

5. Eringen, A. C, simple microfluids, Int. J. Eng. Sci. 2 (1964) 205-217.

6. Eringen, A. C, theory of micropolar fluid, J. Math. Mech., 16 (1966) 1-18.

7. Peddieson, J., McNitt, R. P, Boundary layer theory of micropolar fluid, Recent Adv. Eng. Sci., 5 (1970) $405-$ 426.

8. Ahmadi, G., self-similar solution of incompressible micropolar boundary layer flow over a semi-infinite plate, Int. J. Eng. Sci., 14 (1976) 639.

9. El-Arabawy, H. A. M, effect of suction /injection on the flow of a micropolar fluid past a continuously moving plate in the presence of radiation, Int. J. Heat and Mass Transfer, 46 (2003) 1471-1477.

10. Aziz A, Ali Y, Aziz T, Heat Transfer Analysis for stationary Boundary Layer Slip Flow of a Power-Law Fluid in a Darcy Porous Medium with plate Suction/Injuction, Siddique J.I., (2015). 
11. S. E. E. Hamza, The effect of Suction and Injection on MHD Flow Between Two Porous Concentric Cylinders Filled with Porous Medium, J. Advance in physics 16(2019)

12. Olanrewaju P. O., Makinde O. D.,Effect of thermal diffusion and diffusion thrmo on chemically reacting MHD boundary layer flow of heat and mass transfer past a moving vertical plate with suction / injection, Arabian J. of Science and Engineering, 36(2011) 337-380 .

13. Das, S.S., Satapathy, A., Das J. K., and panda, J.P., Mass transfer effects on MHD flow and heat transfer past a vertical porous plate through a porous medium under oscillatory suction and heat source, Int. J. Heat Mass Transfer, 52(2009), 5962-5969.

14. Das, S.S., Tripathy, U.K., and Das,J.K., Hydromagnetic convective flow past a a vertical porous plate through a porous medium with suction and heat source, Int. J. of Energy and Environment, 1(2010), 467-478 . 